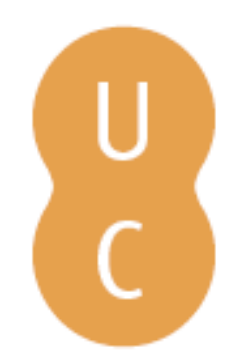

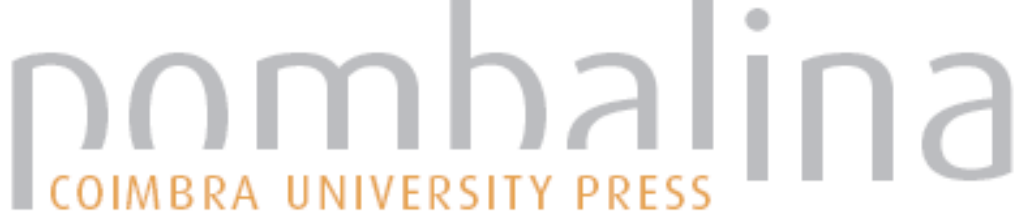

\section{Vulnerabilidade do sistema aquífero na captação da Fadagosa de Nisa (Alentejo,} Portugal): aplicação do Índice Drastic

\author{
Autor(es): $\quad$ Pais, M. F. A. Mota; Antunes, I. M. H. R.; Albuquerque, M. T. D. \\ Publicado por: Imprensa da Universidade de Coimbra \\ URL \\ persistente: URI:http://hdl.handle.net/10316.2/31476 \\ DOI: $\quad$ DOI:http://dx.doi.org/10.14195/978-989-26-0534-0_41 \\ Accessed : $\quad$ 26-Apr-2023 11:12:02
}

A navegação consulta e descarregamento dos títulos inseridos nas Bibliotecas Digitais UC Digitalis, UC Pombalina e UC Impactum, pressupõem a aceitação plena e sem reservas dos Termos e Condições de Uso destas Bibliotecas Digitais, disponíveis em https://digitalis.uc.pt/pt-pt/termos.

Conforme exposto nos referidos Termos e Condições de Uso, o descarregamento de títulos de acesso restrito requer uma licença válida de autorização devendo o utilizador aceder ao(s) documento(s) a partir de um endereço de IP da instituição detentora da supramencionada licença.

Ao utilizador é apenas permitido o descarregamento para uso pessoal, pelo que o emprego do(s) título(s) descarregado(s) para outro fim, designadamente comercial, carece de autorização do respetivo autor ou editor da obra.

Na medida em que todas as obras da UC Digitalis se encontram protegidas pelo Código do Direito de Autor e Direitos Conexos e demais legislação aplicável, toda a cópia, parcial ou total, deste documento, nos casos em que é legalmente admitida, deverá conter ou fazer-se acompanhar por este aviso.

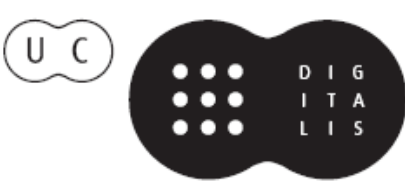



41

\title{
VULNERABILIDADE DO SISTEMA AQUÍFERO NA CAPTAÇÁO DA FADAGOSA DE NISA (ALENTEJO, PORTUGAL): APLICAÇÃO DO INDICE DRASTIC
}

\author{
AQUIFER SYSTEM'S VULNERABILITY FOR \\ FADAGOSA-NISA'S CATCHMENT (ALENTEJO, \\ PORTUGAL) USING DRASTIC INDEX
}

M. F. A. Mota Pais' ${ }^{1}$ I. M. H. R. Antunes ${ }^{1} \&$ M. T. D. Albuquerque ${ }^{1}$

Resumo - As termas da Fadagosa de Nisa, localizadas no nordeste Alentejano, possuem uma relevante importância na economia desta regiáo. Estáo inseridas numa regiáo hidrogeológica de fraca aptidão aquífera, onde predominam granitos fraturados, com fendas preenchidas por material argiloso. A circulação da água é característica de meios fissurados, sendo abastecidas por um único furo, com uma produtividade de 2 1/s. Estão localizadas numa regiáo em que predomina a agricultura, o que lhe confere uma vulnerabilidade para a contaminação. A avaliação da vulnerabilidade de águas subterrâneas é fundamental nos processos de tomada de decisão, visando o ordenamento do território e preservação dos recursos da regiáo. $\mathrm{O}$ objetivo deste trabalho consiste na avaliação espacial da vulnerabilidade da água subterrânea na envolvência da captação da Fadagosa de Nisa. Para tal, foi utilizado o índice de vulnerabilidade Drastic, recorrendo-se ao software ArcGIS para o tratamento da informação e elaboração de cartografia de vulnerabilidade.

Os valores obtidos na aplicação do índice Drastic sugerem uma vulnerabilidade baixa a elevada $(\mathrm{V}=67-153)$. Contudo, a maioria da área apresenta vulnerabilidade baixa (V = 67-119), enquanto as áreas de vulnerabilidade moderada $(\mathrm{V}=119-149)$ correspondem a manchas de pequenas dimensóes, dispersas pela zona de estudo. A zona envolvente da captaçáo da Fadagosa de Nisa apresenta vulnerabilidade baixa, pois ocorre em formaçóes graníticas com um denso sistema de falhas associado e a profundidade do nível freático $(65 \mathrm{~m})$, possivelmente dificultam a infiltração de potenciais elementos poluentes.

\footnotetext{
1 Instituto Politécnico de Castelo Branco, Quinta da Senhora de Mércules, 6001-909 Castelo Branco, Portugal; imantunes@ipcb.pt
} 
Palavras-chave - Sistema aquífero; Termas de Nisa; Vulnerabilidade; Índice Drastic; Cartografia de vulnerabilidade

\begin{abstract}
The thermal catchment of Fadagosa-Nisa is located at Alentejo and has a relevant importance in the economy of this region. Geologically it is predominantly composed of fractured granites, with fractures often filled with clay materials. The occurrence and movement of groundwater is mainly controlled by these fractures and other discontinuities. The study catchment is characterized by low immediate yields $(2 \mathrm{l} / \mathrm{s})$ and located in a region where agriculture is the main economic activity which represents a potential contamination risk. Groundwater vulnerability assessment is crucial in decision-making processes, aiming resource conservation planning. The core objective of this study is to assess the spatial vulnerability related to the groundwater catchment of Fadagosa-Nisa thermal area. The vulnerability was estimated using Drastic index and appropriate ArcGIS software's tools for information processing and preparation of vulnerability maps. The Drastic index indicates a low to high $(V=67-153)$ vulnerability. However, most of the area presents a low vulnerability $(V=67-119)$. Moderate vulnerability $(V=119-149)$ correspond to small areas dispersed across the study area. The Fadagosa-Nisa thermal area occurring in granitic formations with a dense system of faults, associated with the water deep $(65 \mathrm{~m})$, has a low vulnerability to the potential contaminants infiltration.
\end{abstract}

Keywords - Aquifer system; Thermal Nisa area; Vulnerability; Drastic Index; Vulnerability mapping

\title{
1 - Introdução
}

A importância das termas da Fadagosa de Nisa na vida e economia desta região remonta ao século XVIII, com as primeiras edificaçóes datadas do ano de 1792 (FIGUEIREDO, 1956). Contudo, a atividade termal nem sempre esteve ativa ao longo dos anos, tendo registado períodos de desenvolvimento intenso, intercalados por outros de declínio, até situaçôes de abandono. O primeiro contrato de exploração, entre a Direcção-Geral de Geologia e Minas e a Câmara Municipal de Nisa, para exploração da água mineral natural, foi celebrado no ano de 1992 (DIÁRIO DA REPÚBLICA, 1992a). Neste mesmo ano, a portaria no 948/92 define os perímetros de proteção para esta captação (zona imediata, intermédia e alargada), com o objetivo de a proteger, permitindo uma exploração e aproveitamento adequados (DIÁRIO DA REPÚBLICA, 1992b). No ano 2000, foi criada a empresa municipal Ternisa-E.M., com o objetivo de dirigir a exploração das termas. Face à importância deste empreendimento para a economia local e regional, e considerando que a água é um recurso escasso, torna-se imprescindível a adoção de medidas de proteção e de ordenamento adequadas à realidade em estudo.

A vulnerabilidade de um sistema pode ser entendida como a maior ou menor capacidade de atenuação das camadas superficiais do aquífero face à passagem de poluentes, sendo uma propriedade intrínseca do sistema hidrogeológico (PARALTA et al., 2005). A vulnerabilidade intrínseca de um aquífero representa a sua capacidade para receber e transmitir substâncias potencialmente contaminantes, no espaço e ao longo do tempo. Esta dependerá da capacidade de infiltração no meio, do tempo de deslocação da água 
e/ou contaminante da zona não saturada até à saturada, da dinâmica do fluxo da água subterrânea e/ou contaminante e da concentração do contaminante, resultando a capacidade de atenuação do aquífero (CIVITA \& MAIO, 2004). A vulnerabilidade específica, por sua vez, define a sensibilidade da água subterrânea a um contaminante, em função das suas propriedades e das características intrínsecas do sistema aquífero (WANG et al., 2007). Neste caso, a avaliação deve ser feita tendo em atenção as características físicas e químicas do contaminante, tipo de fonte, quantidade e taxa de aplicação (CIVITA \& MAIO, 2004).

O risco de poluição de um sistema aquífero depende da sua vulnerabilidade e da presença de contaminantes em quantidade significativa (RHAMAN, 2008). Deste modo, pode concluir-se que é possível a existência de aquíferos com uma vulnerabilidade eleva$\mathrm{da}$, mas um baixo risco de poluição, devido à ausência de cargas poluentes, ou à presença de um risco de poluição elevado, apesar da baixa vulnerabilidade (LOBO FERREIRA, 1998). Com a delimitação de níveis de vulnerabilidade nos sistemas aquíferos é possível identificar áreas de maior potencial de contaminação e a elaboração de mapas de vulnerabilidade (AL-ZABET, 2002). A avaliação da vulnerabilidade de águas subterrâneas é fundamental nos processos de tomada de decisão, visando o ordenamento do território e preservação dos recursos da região. O objetivo deste trabalho consiste na avaliação da vulnerabilidade da água subterrânea na envolvência da captação da Fadagosa de Nisa (furo ACP4), com recurso ao índice de vulnerabilidade Drastic, e sua posterior representação espacial, utilizando ferramentas adequadas de SIG (ArcGIS). Os mapas obtidos constituem uma cartografia da vulnerabilidade na região em estudo.

\section{2 - Metodologia drastic}

$\mathrm{Na}$ determinação da vulnerabilidade de um sistema aquífero foram desenvolvidas várias metodologias, passando pela construção de índices e aplicação de métodos estatísticos, para a caracterização de grandes áreas de estudo, e ainda metodologias baseadas na simulação de modelos, para aplicação em áreas mais pequenas (WANG et al., 2007). Apesar das várias metodologias existentes, não existe forma satisfatória de representar a vulnerabilidade dos aquíferos, face à dificuldade em integrar todos os parâmetros que influenciam o comportamento dos contaminantes (PARALTA et al., 2005). A construção de índices de síntese, por sua vez, permite considerar conjuntamente a influência de vários fatores que interferem na avaliação da vulnerabilidade do aquífero, constituindo o índice "DRASTIC" uma das metodologias mais utilizadas nesta avaliação (SAMAKE et al., 2011). Este método é de relativa facilidade de aplicação, face à quantidade de informação disponível e possibilidade de aplicação em áreas com diferentes características, com a obtenção de resultados interpretáveis em concordância com um modelo internacionalmente reconhecido (LIGGETT \& ALLEN, 2010).

O índice de vulnerabilidade DRASTIC consiste no somatório da média ponderada de 7 parâmetros (ou indicadores hidrogeológicos) de uma determinada área: profundidade da zona não saturada (D); recarga do aquífero (R); material do aquífero (A); tipo de solo $(\mathrm{S})$; topografia $(\mathrm{T})$; influência da zona vadosa (I) e condutividade hidráulica (C) (ALLER et al., 1987). Cada um destes parâmetros é subdividido em classes representativas, ao qual é atribuído um índice (i), que pode variar entre 1 e 10, de modo a obter-se 
uma correspondência com as características hidrogeológicas locais (valores mais elevados representam uma maior vulnerabilidade). Posteriormente, esses valores são multiplicados pela proporção (p) relativa de cada parâmetro (i) (valor pré-determinado que representa a sua importância relativa na determinaçáo da vulnerabilidade, e que varia entre 1 e 5), e, por fim, obtém-se o valor de vulnerabilidade através do somatório dos diversos produtos obtidos: V = Di x Dp + Ri x Rp +Ai x Ap + Si x Sp + Ti x Tp + Ii x Ip + Ci x Cp. O índice de vulnerabilidade pode variar entre um valor mínimo de 23 (pouco vulnerável) e um valor máximo de 230 (muito vulnerável) (ALLER et al., 1987; LIGGETT \& ALLEN, 2010).

\section{3 - Localização e caracterização da área de estudo}

As termas da Fadagosa de Nisa, com uma área de concessão com 74,88 Ha, localizam-se no concelho de Nisa, inserido na região do Alto Alentejo, distrito de Portalegre, a cerca de $8,8 \mathrm{~km}$ a SSW da vila de Nisa e a $1,1 \mathrm{~km}$ do marco geodésico da Lage da Prata (CMN, 2010).

O concelho de Nisa insere-se no Maciço Hespérico, mais precisamente na Zona Centro Ibérica, sendo dominado pelo Maciço Nisa-Albuquerque, que intruiu e metamorfizou os xistos do grupo das Beiras e os sedimentos do Paleozóico (LNEG, 2010). Associado aos eventos tectónicos locais, no decurso da fase tardia da orogenia Varisca, surgiu um conjunto de falhas que afetaram as rochas pré-existentes, com direção de compressão máxima N-S, e um sistema de falhas conjugado, NNE-SSW, de movimentação esquerda, e direção NNW-SSE, com movimentação direita (LNEG, 2010). De referir, ainda, a ocorrência de movimentos alpinos, que provocaram o rejogo de falhas e fraturas originadas na orogenia Varisca, facilitando o preenchimento silicioso da maioria delas (CMN, 2010). Atravessando os granitos e as rochas metamórficas, ocorrem estruturas filoneanas de quartzo, aplito-pegmatitos e microgranitos, orientados N-S. A fraturação intensa a que o quartzo foi sujeito promoveu o preenchimento das suas fendas com quartzo secundário, acompanhado por mineralizaçóes uraníferas (CATRAPONA et al., 2009).

$\mathrm{Na}$ zona de emergência da água da Fadagosa de Nisa ocorrem extensos afloramentos graníticos, com disjunção esferoidal, sugerindo algum grau de alteração. O sistema de falhas e diaclases apresentam uma direção predominante NE-SW a N50E, subverticais, intersetado por uma falha de sentido N-S, a longo da qual se instalou um pequeno curso de água, encontrando-se a nascente na interseção destas duas falhas (MOREIRA, 1985).

A nível geomorfológico, está inserida na plataforma do Alto Alentejo ou peneplanície do Alto Alentejo, a uma altitude entre 300-320 m, estando as termas de Nisa, numa área aplanada (240 a 310 m), e a captação localizada a cerca de $280 \mathrm{~m}$ de altitude. O declive apresenta valores inferiores a 9\% (MOTA PAIS, 2011), sendo possível verificar a existência de uma suave inclinação para este, em direção à falha do Ponsul (LNEG, 2010).

A área de estudo está inserida na bacia hidrográfica do rio Tejo, onde ocorrem 5 sub-bacias hidrográficas, das quais quatro drenam na direção SE-NW para afluentes do rio Tejo e uma drena na direçáo NE-SW, para a ribeira do Sôr, afluente do rio Sorraia (MOTA PAIS, 2011). De um modo geral, o regime de escoamento é temporário, localmente com comportamento torrencial, excetuando-se os rios Tejo e Sever, que apresentam um regime permanente (CMN, 2010). As características hidrogeológicas dependem das 
litologias presentes, de natureza granítica na parte sul e metassedimentar na parte norte, com solos pouco desenvolvidos, que derivam das rochas xistentas e apresentam uma constituição argilosa (CATRAPONA et al., 2009). No concelho de Nisa, é possível obter uma produtividade hidrológica entre os $0,5 \mathrm{l} / \mathrm{s}$ e $1,0 \mathrm{l} / \mathrm{s}$ na zona de granito, e de $1.0 \mathrm{l} / \mathrm{s}$ e $1.5 \mathrm{l} / \mathrm{s}$ na zona de xisto. A menor produtividade hidrogeológica do granito relaciona-se com um processo de diaclasamento e fraturação menos intenso e com o preenchimento das fendas por argila, dificultando a circulação subterrânea (CMN, 2010). Estas características permitem que se divida o concelho em duas grandes formaçôes com fraca aptidão aquífera: 1. o setor aquífero pouco produtivo Amieira - Montalvão, constituído por rochas metamórficas, conjuntamente com uma crista quartzítica (Serra de S. Miguel) e uma faixa de corneana; 2. o setor aquífero das rochas ígneas (ou granitos) de Nisa, Portalegre e Santa Eulália, onde se insere a área de estudo, cujas falhas e fraturas promovem um escoamento subterrâneo, característico de meios fissurados (CMN, 2010). A recarga do aquífero é feita por infiltração direta da precipitação e por influência dos cursos de água superficiais, estimando-se que a taxa média de recarga na zona se situe em cerca de 106,0 mm/ano (CMN, 2010).

Nas zonas graníticas não é muito abundante a ocorrência de nascentes e, no caso da Fadagosa de Nisa, existe uma única nascente com um baixo caudal, sugerindo uma fraca meteorização da rocha granítica. Com a perfuração em profundidade, na zona das termas, a $65 \mathrm{~m}$ de profundidade (furo ACP4), foi possível obter um caudal 40 vezes superior ao caudal da nascente, com uma produtividade de 2 1/s, mantendo uma boa estabilidade composicional da água ao longo do ano (CALADO, 2001). As águas da nascente da Fadagosa de Nisa estão condicionadas pela ascensão ao longo de interseção das falhas que originam esta emergência, estando expressa através de numerosas fraturas e de uma ampla caixa de falha.

No Concelho de Nisa, é possível identificar duas unidades pedológicas principais: os litossolos, no norte do concelho, correspondendo a solos incipientes, de espessura reduzida, associados a formaçôes xisto-grauváquicas, e os solos litólicos não húmicos, a sul, onde ocorre a área de estudo, sendo pouco evoluídos e delgados, de relevo aplanado, em formaçôes graníticas. Pode referir-se que predominam solos com capacidade de uso muito baixa e baixa, associada a elevado risco de erosão, limitando o solo para atividades agrícolas e destinando-os preferencialmente para aproveitamentos florestais ou pastagens naturais (CMN, 2010). Na área das Termas da Fadagosa de Nisa e respetivas zonas de proteção, predominam as áreas agrícolas heterogéneas (MOTA PAIS, 2011).

\section{4 - Avaliação da vulnerabilidade na região da captação da Fadagosa de Nisa}

A vulnerabilidade do sistema aquífero da captação das termas da Fadagosa de Nisa foi avaliada através do cálculo do índice de vulnerabilidade DRASTIC, desenvolvido por ALLER et al. (1987). Para o cálculo do índice DRASTIC foram quantificados os respetivos parâmetros e correspondentes ponderaçóes em 198 poços georreferenciados, e interpolados, para a área em estudo, recorrendo ao algoritmo Inverse Distance Weighted (IDW). Recorreu-se ao software ArcMAP 9.3.1 da ESRI, tendo por base o sistema de coordenadas “Datum 73; Hayford Gauss; IPCC”, para o processamento dos atributos e cartografia de vulnerabilidades. 


\section{1 - Determinação dos parâmetros do Índice DRASTIC}

A captação das termas da Fadagosa de Nisa está localizada na zona correspondente ao setor pouco produtivo dos granitos de Nisa (Fig. 1). A superfície interpolada, obtida para a profundidade da zona saturada (D), indica valores médios de profundidade superiores a 30,45m, correspondendo ao índice 1 (menos vulnerável) (Fig. 2). A recarga média (R) para a zona de granito de Nisa é de 106 mm/ano (CMN, 2010), sendo-lhe atribuído um valor igual a 6 (MOTA PAIS, 2011).



Fig. 1 - Localização da captação das Termas da Fadagosa de Nisa e delimitação da área de estudo para aplicaçâo vulnerabilidade "DRASTIC".

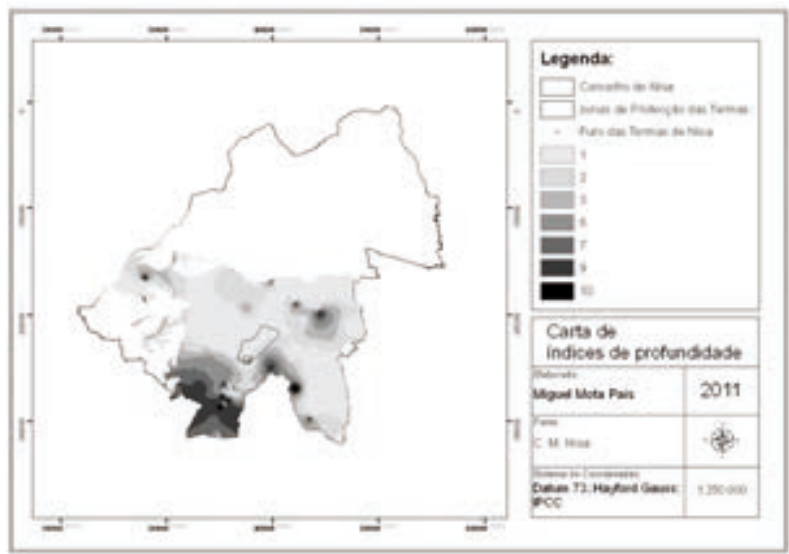

Fig. 2 - Carta interpolada para o atributo profundidade (D).

$\mathrm{Na}$ área de estudo predominam as rochas graníticas, para as quais pode ser atribuído uniformemente em profundidade o valor 4 (A). Relativamente ao tipo de solo que ocorre na 
área de estudo, foram atribuídos valores de acordo com a escala do índice DRASTIC, predominando os solos litólicos, aos quais é atribuído um valor 9 (S) (Fig. 3). Nos locais em que se verifica a existência de vários tipos de solos, foi selecionado o que representa uma maior percentagem de ocupação (MOTA PAIS, 2011). Para atribuição dos valores relativos à topografia (T), e tendo em consideração que a área em estudo é essencialmente plana, os valores considerados são aproximados, oscilando essencialmente em torno de 5, 9 e 10, correspondendo a declives mais suaves. Apenas na zona oeste da área de estudo ocorrem declives mais acentuados, localizados próximo do rio Tejo (Fig. 4). Para a influência da zona vadosa (I), consideram-se os dados relativos ao material do aquífero, pelo que, devido às características litológicas desta região, os resultados são semelhantes sendo assumida uma condutividade hidráulica média, para as formaçóes graníticas, de $3,2 \times 10^{-6} \mathrm{~m} / \mathrm{s}$, e para as formaçóes meta-

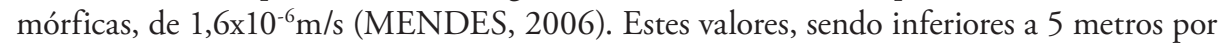
dia, correspondem, para as duas situaçóes, ao valor 1 para o atributo (C) (Figura 5).



Fig. 3 - Carta de índices do tipo de solo (S).

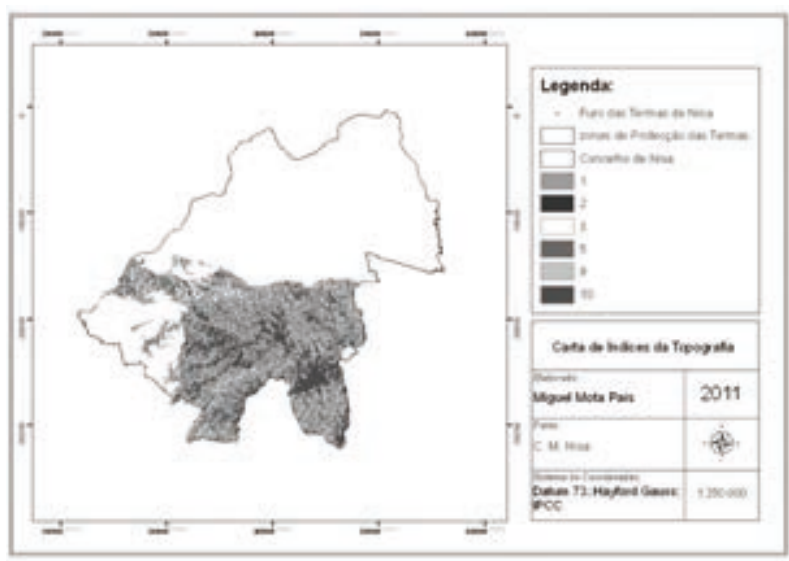

Fig. 4 - Carta de índices da topografia (T). 




Fig. 5 - Carta de índices da condutividade Hidráulica (C).

\section{2 - Mapa de vulnerabilidade}

Como resultado do somatório ponderado, de acordo com a metodologia DRASTIC, dos diversos mapas, relativos a cada um dos atributos, obteve-se a cartografia da vulnerabilidade intrínseca na área de estudo (Figura 6). Apesar de os valores de vulnerabilidade (V) variarem entre 67 e 153, a maioria da área de estudo apresenta baixa vulnerabilidade, com valores entre 67 e 119 (Figura 6). Nas áreas com vulnerabilidade moderada, verifica-se a existência de manchas de pequenas dimensóes dispersas, com uma de maiores dimensóes a SW da captação da Fadagosa de Nisa, apresentando valores entre 119 e 149, estando geralmente associada à existência de captações de água (Figura 6).



Fig. 6 - Carta de vulnerabilidade DRASTIC.

Relativamente à captação das termas da Fadagosa de Nisa e respetivas zonas de proteção (imediata, intermédia e alargada), situam-se numa classe de vulnerabilidade baixa 
(Fig. 6). A localização do furo da captação numa falha e a superfície piezométrica a $65 \mathrm{~m}$ representam, seguramente, uma forte influência nos resultados obtidos. Os restantes atributos, apesar das suas características individuais, em conjunto apresentam um contributo reduzido na quantificação da vulnerabilidade.

\section{5 - Conclusão}

A determinação do índice de vulnerabilidade intrínseca das águas subterrâneas na envolvência da captação da Fadagosa de Nisa, através do cálculo do índice DRASTIC, permite concluir que:

- na zona envolvente desta captação, a vulnerabilidade é baixa apesar de existirem zonas com vulnerabilidade alta e moderada, face a um conjunto de características relacionadas com a geologia da regiáo e profundidade do nível freático, que podem dificultar a infiltração de possíveis elementos poluentes;

- apesar de uma possível contaminação na área não ser expectável, é importante referir que, em relação a outras zonas, com uma vulnerabilidade intrínseca superior, há que considerar sempre um risco associado superior.

A avaliação da vulnerabilidade de águas subterrâneas é fundamental nos processos de tomada de decisão, visando o ordenamento do território e preservaçáo dos recursos da regiâo; pelo que se torna imperativa a tomada de medidas preventivas e de monitorização ao longo do tempo.

\section{Referências Bibliográficas}

AL-ZABET, T. (2002) - Evaluation of aquifer vulnerability to contamination potential using the DRASTIC method. Env. Geol., 43, p. 203-208.

ALLER, L., BENNETT, T., LEHR, J. H., PETTY, R. H. \& HACKETT, G. (1987) - DRASTIC: a standardised system for evaluating groundwater pollution potential using hydrogeologic settings. USEPA Report 600/2- 87/035, Robert S. Kerr; Environmental Research Laboratory; Ada, Oklahoma.

CALADO, C. M. A. (2001) - A ocorrência de água sulfúrea alcalina no Maciço Hespérico: quadro hidrogeológico e quimiogénese. Dissertação para obtençấo do grau de Doutor em Geologia, Especialidade de Hidrologia (Não publicado). Departamento de Geologia da Faculdade de Ciências da Universidade de Lisboa.

CMN - CÂMARA MUNICIPAL de NISA (2010) - Revisão do Plano Director Municipal - Caracterização do território Municipal, p. $54-68$.

CATRAPONA, A., LOURENÇO, C., MIDÓES, C., OliVEIRA, D., CARVALHO, J. \& FALÉ, P. (2009) - Contributo para a Revisão do PDM de Nisa - Geologia e Recursos Geológicos.

CIVITA, M. \& MAIO, M. D. (2004) - Assessing and mapping groundwater vulnerability to contamination: Italian "Combined” Approach. Geofísica internacional, 43, p. 513-532.

DIÁRIO DA REPÚBLICA (1992a) - D.R. III Série, nº17 de 21-01-92. Extracto do contracto de concessão das Termas da Fadagosa de Nisa. Imprensa Nacional da Casa da Moeda.

DIÁRIO DA REPÚBLICA (1992b) - Portaria 948/92 de 29-09-1992. Define os perímetros de segurança para a captação das Termas da Fadagosa de Nisa. Imprensa Nacional da Casa da Moeda. 
FIGUEIREDO, J. F. (1956) - Monografia de Nisa. Imprensa Nacional Casa da Moeda; Câmara Municipal de Nisa.

LNEG - LABORATÓRIO NACIONAL DE ENERGIA E GEOLOGIA (2010) - Recursos Geológicos e Hidrológicos do município de Nisa - relatório técnico. Acordo de Cooperação entre a Câmara Municipal de Nisa e Laboratório Nacional de Energia e Geologia. Relatório Final da Fase B.

LIGGETT, J. E. \& ALLEN, D. M. (2010) - Evaluating the sensitivity of DRASTIC using the different data sources; interpretations and mapping approaches. Environ Earth sci, doi: 10.1007/s 12665-010-0642-z.

LOBO-FERREIRA, J. P. C. (1998) - Vulnerabilidade à poluição de águas subterrâneas: fundamentos e conceitos para uma melhor gestão e protecção dos aquíferos de Portugal. 4º Congresso da Água, Lisboa.

MENDES, E. (2006) - Perímetros de protecção de captações de água subterrânea para consumo humano em zonas de montanha: Caso de estudo da cidade da Covilhã. Dissertação apresentada para a obtenção do grau de Mestre em Geotecnia Ambiental (Não publicado). Universidade da Beira Interior.

MOREIRA, J. D. D. (1985) - Relatório dos testes de prospecção geoeléctrica efectuados na área da nascente minero-medicinal da Fadagosa de Nisa. Direcção Geral de Geologia e Minas.

MOTA PAIS, M. F. A. (2011) - Avaliação da vulnerabilidade do sistema aquífero da captação da Fadagosa de Nisa (Concelho de Nisa). Dissertação apresentada para obtenção do grau de Mestre em Monitorização de Riscos e Impactes Ambientais (Não publicado). Instituto Politécnico de Castelo Branco.

PARALTA, E. A., FRANCES, A. P. \& RIBEIRO, L. F. (2005) - Avaliação da vulnerabilidade do sistema aquífero dos Grabos de Beja e análise crítica das redes de monitorização no contexto da directiva da água. VII Simpósio de Hidráulica e Recursos Hídricos dos Palop`s (silusba). Évora.

RAHMAN, A. (2008) - A GIS based DRASTIC model for assessing groundwater vulnerability in shallow aquifer in Aligarh, India. Appl. Geog., 28, p. 32-53.

SAMAKE, M., TANG, Z., HLAING, W., NDOH, M. I., KASEREKA, K. \& BALOGUN, W. O. (2011) Groundwater Vulnerability Assessment in Shallow Aquifer in Linfen Basin, Shanxi Province, China Using DRASTIC Model. Journal of Sustainable Development, 4, No. 1.

WANG, Y., MERKEL, B. J., LI, Y., YE, H., FU, S. \& IHM, D. (2007) - Vulnerability of groundwater in Quaternary aquifers to organic contaminants: a case study in Wuhan City, China. Env. Geol. 53, p. 479-484. 